4. Lee AYS, Cassar PM, Johnston AM, Adelstein S. Clinical use and interpretation of serum protein electrophoresis and adjunct assays. $\mathrm{Br} J$ Hosp Med 2017; 78: C18-20.

5. Finn WG, Gulbranson R, Fisher S, et al. Detection of polyclonal increases in immunoglobulin G4 subclass by distinct patterns on capillary serum protein electrophoresis: diagnostic pitfalls and clinical observations in a study of 303 cases. Am J Clin Pathol 2016; 146: 303-11.

6. Ebbo M, Grados A, Bernit E, et al. Pathologies associated with serum IgG4 elevation. Int J Rheumatol 2012; 2012: 602809.

7. Masaki Y, Kurose N, Yamamoto M, et al. Cutoff values of serum IgG4 and histopathological IgG4+ plasma cells for diagnosis of patients with IgG4-related disease. Int J Rheumatol 2012; 2012: 580814.

DOI: https://doi.org/10.1016/j.pathol.2021.05.092

\section{Cutaneous clear cell sarcoma with an epidermal component mimicking melanoma}

Sir,

Malignant cutaneous tumours composed of spindled and epithelioid cells often present a diagnostic challenge, with a range of differential diagnoses including keratinocytic and adnexal carcinomas, melanoma and various sarcomas. Morphological clues should be sought to aid correct diagnosis, sometimes supplemented by the judicious use of immunohistochemistry or even molecular techniques. The presence of an epidermal component is usually interpreted as evidence of a precursor lesion in cases of melanoma or keratinocytic carcinoma and helps guide selection of further ancillary tests. Here, we present a case of primary cutaneous clear cell sarcoma with an intraepidermal component, initially considered to represent a nodular melanoma. To the best of our knowledge, this is the first reported case of cutaneous clear cell sarcoma exhibiting prominent Pagetoid spread, closely mimicking melanoma and highlighting the potential diagnostic pitfall of relying on intraepidermal growth patterns for the diagnosis of cutaneous tumours.

A 30-year-old man presented with an ulcerated nodular cutaneous lesion on the distal third toe, which had been present for several years. Excision revealed a $21 \mathrm{~mm}$ nodule eroding into bone (Fig. 1A) which histologically demonstrated a nested pleomorphic spindled and epithelioid cell tumour containing occasional multinucleated giant cells (Fig. 1B). Tumour cells had abundant amphophilic cytoplasm without discernible pigmentation (Fig. 1C). A nested junctional component was present, as well as single tumour cells showing Pagetoid spread (Fig. 1D) highlighted on immunohistochemistry for S100 (Fig. 1E). The tumour was also SOX10 positive with focal positivity for HMB45 at its deep and peripheral aspects (Fig. 1F,G). Immunostains for cytokeratins, Melan A, SMA and desmin were negative. The tumour was initially considered to represent a T4b nodular melanoma.

The tumour recurred and the patient developed regional nodal metastasis in six of 14 inguinal nodes. Mutation testing of $B R A F$ and NRAS were negative, and he was commenced on adjuvant nivolumab. However, the patient recurred on treatment, developing pulmonary and bone metastases. An escalated regimen of combined ipilimumab/nivolumab was poorly tolerated, with the patient developing severe, immunerelated hepatitis. On further disease progression, he was treated with multiple lines of systemic therapy, including low dose ipilimumab, combination nivolumab and lenvatinib, and combination carboplatin and paclitaxel. Tumour tissue was sent for comprehensive targeted mutation testing through the Australian Genomic Cancer Medicine Centre's Molecular Screening and Therapeutics (MoST) Study which identified an EWSR1-ATF1 gene fusion and a TERT promoter mutation. ${ }^{1}$ In light of the new molecular findings, the original pathology was reviewed and considered to represent an EWSR1-ATF1 fusion positive cutaneous clear cell sarcoma with an epidermal component. Crizotinib was initiated, but subsequent positron emission tomography/computed tomography scans showed disease progression.

Clear cell sarcoma was initially described as a deepseated tumour arising from tendons and aponeuroses, with a predilection for the lower limbs of young patients. ${ }^{2}$ Given the morphological similarities, stainable intracytoplasmic melanin pigment, diffuse S100 expression and the presence of ultrastructural melanosomes, clear cell sarcoma is sometimes referred to as 'melanoma of soft parts', and thought to be derived from the neural crest. Early case series found that the tumour rarely involved the subcutis or dermis, leading the authors to conclude that an absence of epidermal component or history of melanoma could strongly favour a diagnosis of clear cell sarcoma over melanoma. ${ }^{3-5}$

First reported in 2009, there have now been 30 reported cases of primary cutaneous (dermal) clear cell sarcoma. ${ }^{6-8}$ The vast majority of cutaneous presentations showed typical morphological features of clear cell sarcoma; however, many of these features overlap with those of melanoma. Multinucleated floret-like giant cells are a characteristic feature of clear cell sarcoma and can be diagnostically helpful when present, but are not seen in all cases. Occasionally the tumour may expand the dermal papillae and encroach on the dermoepidermal junction. If this occurs, there is frequent discohesion and clefting artefact around individual nests, closely resembling an intraepidermal component. ${ }^{6,8}$ There have only been two reported cases of cutaneous clear cell sarcoma where single cell lentiginous growth along the junctional epidermal zone has been noted. ${ }^{6,9}$ To the best of our knowledge, our case here is the first to demonstrate prominent Pagetoid spread within the epidermis, closely mimicking an in situ component of primary melanoma. The extensive ulceration is also a unique feature of our case, suggesting a greater degree of epidermal destruction than previously described in clear cell sarcoma, and adding to the difficulty in distinguishing the tumour from an aggressive melanoma.

Morphological distinction of clear cell sarcoma from melanoma can be aided by appreciating the relative lack of intralesional heterogeneity and cytological atypia within the dermal component in clear cell sarcoma compared to the typical morphology of a thick melanoma. Multinucleated giant cells are more often seen in clear cell sarcoma and, when present in melanoma, usually display a more haphazard distribution of pleomorphic nuclei, rather than the floret-like arrangement of monomorphic nuclei seen in those of clear cell sarcoma. ${ }^{8}$ 

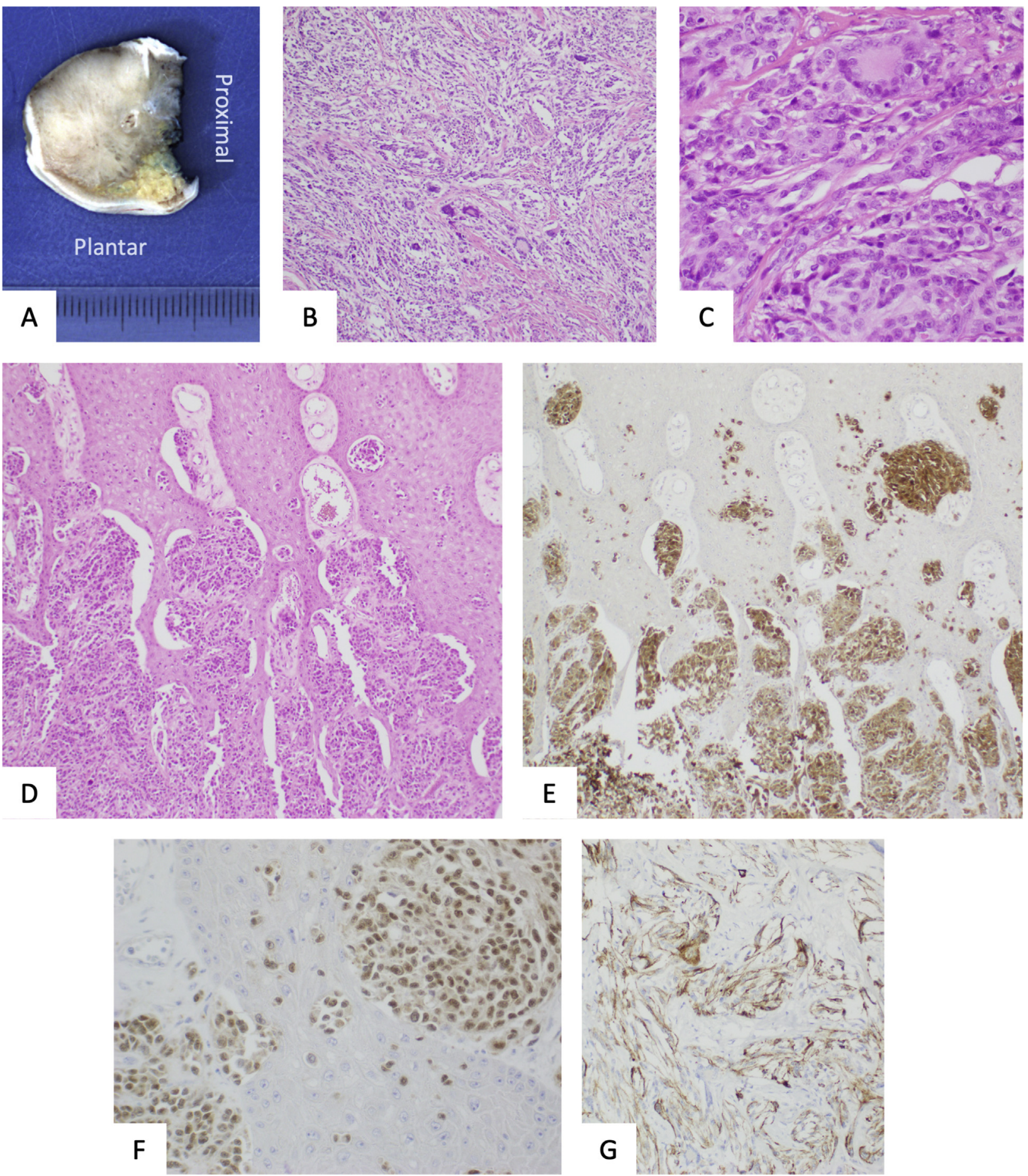

Fig. 1 Lesion of the third distal phalanx. (A) The $21 \mathrm{~mm}$ ulcerated cutaneous tumour centred within the dermis, eroding into bone. (B) Nests of epithelioid and spindled cells separated by fibrous septa, with occasional multinucleated giant cells. (C) The cytoplasm is amphophilic and focally pale and lacks discernible melanin pigment. There are occasional intraepidermal nests and single cells showing Pagetoid spread. (D) Nests of tumour were present at the dermoepidermal junction. (E) Immunohistochemistry for S100 is diffusely positive in junctional nests and Pagetoid cells. (F) SOX10 highlights intraepidermal tumour cells. (G) HMB45 expression was strongest in the deep aspect of the tumour.

Regardless of the morphological, immunohistochemical and ultrastructural overlap between clear cell sarcoma and melanoma, the underlying molecular drivers of tumourigenesis are distinct between the two tumours. Demonstration of EWSR1-ATF1 (and the less common EWSR1-CREB1) fusion is now considered diagnostic of clear cell sarcoma, and testing by fluorescence in situ hybridisation (FISH) or reverse-transcription polymerase chain reaction (RT-PCR) 
should be utilised in ambiguous cases to distinguish clear cell sarcoma from melanoma that usually displays a complex karyotype. ${ }^{4,10}$ Early studies suggested that $B R A F$ mutations are exclusively seen in melanoma. However, rare tumours with morphological features of clear cell sarcoma have been recently shown to harbour concurrent EWSRl fusions and $B R A F \mathrm{~V} 600 \mathrm{E}$ mutations, providing an avenue for targeted treatments in these cases. ${ }^{11,12}$ Whether these tumours represent melanoma rather than clear cell sarcoma is perhaps debatable and probably academic in the context of a poor prognosis tumour harbouring a targeted therapeutic mutation $(B R A F)$.

The case reported here clearly highlights the diagnostic difficulty in distinguishing clear cell sarcoma from melanoma, made more challenging by the cutaneous location, extensive ulceration and intraepidermal growth with Pagetoid spread, not previously described in this disease. This unique presentation broadens the morphological spectrum described in clear cell sarcoma and highlights that intraepidermal growth, previously thought to exclude clear cell sarcoma from diagnostic consideration, can occur. ${ }^{6}$ An awareness of cutaneous presentations of clear cell sarcoma, its salient morphological features, as well as the importance of utilising specific molecular testing when appropriate, should allow for navigation through this diagnostic pitfall.

Acknowledgement: We thank Dr Ivan Canoy for the macroscopic images. We also thank A/Prof Robyn Saw, A/Prof Angela Hong, Dr Vivek Bhadri and the MoST study team. Support from colleagues at Melanoma Institute Australia and Royal Prince Alfred Hospital is also gratefully acknowledged.

Conflicts of interest and sources of funding: AJP is supported by a Fellowship funded by Deborah McMurtrie and John McMurtrie AM through the Melanoma Institute Australia. GVL is supported by an NHMRC Practitioner Fellowship and the University of Sydney Medical Foundation. RAS is supported by a NHMRC Practitioner Fellowship. RAS has received fees for professional services from Provectus Biopharmaceuticals Australia, QBiotics, Novartis, Merck Sharp \& Dohme, NeraCare, Amgen Inc., BristolMyers Squibb, Myriad Genetics and GlaxoSmithKline. GVL is consultant advisor for Aduro Biotech Inc., Amgen Inc., Array Biopharma Inc., Boehringer Ingelheim International GmbH, Bristol-Myers Squibb, Highlight Therapeutics SL, Merck Sharp \& Dohme, Novartis, OncoSec, Pierre Fabre, QBiotics, Regeneron Pharmaceuticals Inc., SkylineDX BV and Specialised Therapeutics Australia Pty Ltd. The remaining authors state that there are no conflicts of interest to disclose.

\section{Alison J. Potter ${ }^{1,2,3}$, Florentia Dimitriou ${ }^{2,4}$, Rooshdiya Z. Karim ${ }^{1,5}$, Annabelle Mahar ${ }^{1}$, Charles Chan ${ }^{5,6}$, Geor- gina V. Long ${ }^{2,5,7}$, Richard A. Scolyer ${ }^{1,2,5}$}

${ }^{1}$ Tissue Pathology and Diagnostic Oncology, Royal Prince Alfred Hospital, and NSW Health Pathology, Sydney, NSW, Australia; ${ }^{2}$ Melanoma Institute Australia, The University of Sydney, Sydney, NSW, Australia; ${ }^{3}$ Faculty of Medicine, University of New South Wales, Sydney, NSW, Australia;
${ }^{4}$ Department of Dermatology, University Hospital Zurich, Zurich, Switzerland; ${ }^{5}$ Faculty of Medicine and Health, The University of Sydney, Sydney, NSW, Australia; ${ }^{6}$ Department of Anatomical Pathology Department, Concord Repatriation General Hospital, and NSW Health Pathology, Sydney, NSW, Australia; ${ }^{7}$ Royal North Shore and Mater Hospitals, Sydney, NSW, Australia

Contact Prof Richard A. Scolyer.

E-mail: richard.scolyer@health.nsw.gov.au

1. Thavaneswaran S, Sebastian L, Ballinger M, et al. Cancer Molecular Screening and Therapeutics (MoST): a framework for multiple, parallel signal-seeking studies of targeted therapies for rare and neglected cancers. Med J Aust 2018; 209: 354-5.

2. Enzinger FM. Clear-cell sarcoma of tendons and aponeuroses. An analysis of 21 cases. Cancer 1965; 18: 1163-74.

3. Kindblom LG, Lodding P, Angervall L. Clear-cell sarcoma of tendons and aponeuroses. An immunohistochemical and electron microscopic analysis indicating neural crest origin. Virchows Arch A Pathol Anat Histopathol 1983; 401: 109-28.

4. Graadt van Roggen JF, Mooi WJ, Hogendoorn PC. Clear cell sarcoma of tendons and aponeuroses (malignant melanoma of soft parts) and cutaneous melanoma: exploring the histogenetic relationship between these two clinicopathological entities. J Pathol 1998; 186: 3-7.

5. Fletcher CD, McKee PH. Sarcomas-a clinicopathological guide with special reference to cutaneous manifestation. IV. Extraskeletal osteosarcoma, extraskeletal chondrosarcoma, alveolar soft part sarcoma, clear cell sarcoma and discussion. Clin Exp Dermatol 1985; 10: $523-39$.

6. Luzar B, Billings SD, de la Fouchardiere A, et al. Compound clear cell sarcoma of the skin - a potential diagnostic pitfall: report of a series of 4 new cases and a review of the literature. Am J Surg Pathol 2020; 44: $21-9$

7. Rodriguez-Martin M, Saez-Rodriguez M, Esquivel B, et al. Clear cell sarcoma: a case mimicking primary cutaneous malignant melanoma. Indian J Dermatol 2009; 54: 168-72.

8. Hantschke M, Mentzel T, Rutten A, et al. Cutaneous clear cell sarcoma: a clinicopathologic, immunohistochemical, and molecular analysis of 12 cases emphasizing its distinction from dermal melanoma. Am J Surg Pathol 2010; 34: 216-22.

9. Kiuru M, Hameed M, Busam KJ. Compound clear cell sarcoma misdiagnosed as a Spitz nevus. J Cutan Pathol 2013; 40: 950-4.

10. Song JS, Choi J, Kim JH, et al. Diagnostic utility of EWS break-apart fluorescence in situ hybridization in distinguishing between noncutaneous melanoma and clear cell sarcoma. Pathol Int 2010; 60: $608-13$.

11. Park BM, Jin SA, Choi YD, et al. Two cases of clear cell sarcoma with different clinical and genetic features: cutaneous type with BRAF mutation and subcutaneous type with KIT mutation. Br J Dermatol 2013; 169: $1346-52$.

12. Hocar O, Le Cesne A, Berissi S, et al. Clear cell sarcoma (malignant melanoma) of soft parts: a clinicopathologic study of 52 cases. Dermatol Res Pract 2012; 2012: 984096.

DOI: https://doi.org/10.1016/j.pathol.2021.05.097

\section{Dedifferentiated melanoma with MDM2 gene amplification mimicking dedifferentiated liposarcoma}

Sir,

We present a 73-year-old female with melanoma that mimicked dedifferentiated liposarcoma (DD-LPS) both morphologically and molecularly in a subclone 